\title{
Pathway-based analysis for genome-wide association study data of bipolar disorder provides new insights for genetic study
}

\section{Dear Editor,}

Bipolar disorder (BD) is an episodic recurrent pathological mood disturbance with estimated heritability ranging from 80\%-85\% (Barnett and Smoller, 2009). Although many studies have indicated that $\mathrm{BD}$ is a polygenic disease influenced by many genes with small effect, the pathogenesis of $B D$ is still not well understood. According to the catalog of published genome-wide association studies (GWAS) (Welter et al., 2014), there have been 31 BD GWASs (including bipolar disorder related symptoms and combined GWAS with other related disorders) till 01/15/2015, including the largest BD GWAS from the Psychiatric Genomics Consortium (PGC) (Sklar et al., 2011). But these GWAS analyses mainly focused on single SNP/gene and only identified a number of significant SNPs that account for a small proportion of the genetic variants. To detect the association between pathway and trait for further GWAS data interpretation and mechanism study, pathway-based analysis (PBA) has been introduced to analyze GWAS data (Wang et al., 2010).

Comparing with traditional genetic association analyses, PBA can detect the additive effects of multiple minor genes. It has been proved to be a feasible solution to interpret GWAS result and promote the analytical level from SNP/gene to pathway. In recent years, a number of PBA methods have been developed whose null hypothesis being tested can be broadly classified as 'self-contained' versus 'competitive' based on whether comparisons were made between genes in a specific pathway and non-associated genes or other genes in the genome. Additionally, the input data of these statistical methods can be broadly classified into two types: SNP $P$-values and raw genotype (Wang et al., 2010).

Among the several published PBA studies for BD (as collected in BDgene (Chang et al., 2013)), the majority of the data sources focused on the Wellcome Trust Case Control Consortium (WTCCC) data (Wellcome Trust Case Control 2007), which was published several years ago. The large scale of PGC data has been used in two PBA studies (Duncan et al., 2014; Yu et al., 2014a), but they are both hypothesis-based and only focus on one type of pathway instead of all pathways. In addition, using more than one
PBA tools to get the consistent result has become more popular (Duncan et al., 2014) although most of the early studies only used one. By now, the results of published PBA studies are still of low repeatability and need further replication. Hence, it is necessary to further analyze the large scale of GWAS data from PGC by using PBA methods to get more reliable disease related pathways.

In this study, we analyzed six BD GWAS datasets from PGC (Sklar et al., 2011) by merging them into two groups according to the platform. The same quality control (QC) process was conducted for both groups of data by using PLINK (see supplementary material for detail). After QC, the two groups of datasets contained 4568 cases and 6255 controls in total, and the numbers of overlapped SNPs were 276,592 in Group A and 477,624 in Group B separately (as shown in Table S1). We used three tools (i-GSEA4GWAS v2 (Zhang et al., 2014), SNP Ration Test (SRT) (O'Dushlaine et al., 2009) and GenGen (Wang et al., 2007)) for our analysis. The brief introduction and parameter selection about these three tools were described in supplementary material.

The pathway-based analysis results for two groups of GWAS datasets by three PBA tools were summarized in Table 1. By inputting SNP $P$-value list, $i$-GSEA4GWAS v2 generated 31 candidate pathways for Group $A$ and 31 candidate pathways for Group B, which shared 12 pathways between Group A and Group B. The calculation of gene-set enrichment score was similar between $i$-GSEA4GWAS v2 and GenGen, but GenGen used genotype data as input and permutation was conducted at phenotype level. GenGen got 133 candidate pathways from Group B, but none from Group A. Furthermore, SRT provided 10 candidate pathways from Group A and 5 candidate pathways from Group B, and there was no overlapped pathway between Group A and Group B. Comparison among different PBA tools for the same GWAS dataset was conducted. For Group A, i-GSEA4GWAS v2 shared four pathways with SRT; no shared pathway for either other pairs of tools and three tools. For Group B, i-GSEA4GWAS v2 shared 27 pathways and two pathways with GenGen and SRT separately; GenGen and SRT shared three pathways, in which, two pathways were shared by three tools. 
Table 1. Comparison result for different groups of GWAS datasets and PBA tools

\begin{tabular}{lccc}
\hline & Group A & Group B & $\begin{array}{c}\text { Overlapped among } \\
\text { Group A and Group B }\end{array}$ \\
\hline$i$-GSEA4GWAS v2 & 31 & 31 & 12 \\
Overlapped in GenGen & 0 & 27 & 2 \\
Overlapped in SRT & 4 & 2 & 0 \\
Overlapped in three tools & 0 & 133 & 3 \\
GenGen & 0 & 27 & 0 \\
Overlapped in SRT & 0 & 2 & 5 \\
Overlapped in $i$-GSEA4GWAS v2 & 0 & 3 \\
Overlapped in three tools & 0 & 2 & \\
SRT & 10 & 2 & \\
Overlapped in GenGen & 0 & 4 & \\
Overlapped in $i$-GSEA4GWAS v2 & 0 & & \\
Overlapped in three tools & & & \\
\hline
\end{tabular}

As a result, 33 unique pathways were obtained from the shared pathways by GWAS datasets or PBA tools. The list of pathways and supported datasets and tools were shown in Table 2. Among these 33 pathways, one pathway "TGF beta signaling pathway" was validated by one PBA tool, two pathways ("Oocyte meiosis" and "Ubiquitin mediated proteolysis") were validated by all three PBA tools, other pathways were validated by two PBA tools. Moreover, six pathways had FDR $<0.05$ in Group $A$ dataset and six pathways had FDR $<0.05$ in Group B dataset. Especially, 'Retinol metabolism' and 'Metabolism of xenobiotics by cytochrome P450' had FDR < 0.05 in both Group A and Group B datasets, which showed great possibility of correlation with $\mathrm{BD}$.

Our analysis also validated several pathways reported by previous PBA studies. For example, phosphatidylinositol signaling system and p53 signaling pathway were identified as risk pathways by Chen et al. using a risk-scoring measurement to fuse SNPs and pathways (Chen et al., 2009); $\mathrm{N}$-Glycan biosynthesis, ABC transporters and cell cycle were identified in (Peng et al., 2010) by using hypergeometric test; Neurotrophin signaling pathway has not been reported by PBA of BD yet, but it has been reported by network analysis paper for schizophrenia (Yu et al., 2014b), and neurotrophic signaling cascades has played an important role in the pathophysiology and treatment of bipolar disorder (Shaltiel et al., 2007).

Besides the pathways validated by other PBA papers, we also identified several novel pathways, which have not been reported to be associated with bipolar disorder by PBA study but have been validated in other literature and deserve further attention, including 'Ubiquitin mediated proteolysis' and 'Oocyte meiosis', which were validated by three PBA tools in our analysis and also statistical significant in Group A dataset (FDR < 0.05); 'Retinol metabolism' and 'Metabolism of xenobiotics by cytochrome P450', which were identified in both two groups with significant association with BD $(P$-value $<0.05$, FDR $<0.05)$. Evidence for the association of these pathways with bipolar disorder and other related psychiatric disorders from literature was summarized in the supplementary material.

In summary, compared with previous PBA studies on $B D$, our analysis includes more samples and uses genotype data to analyze all KEGG pathways. By using multiple GWAS datasets and PBA tools, our analysis could provide better interpretation for the GWASs of BD to further identify disease-related mechanism. The pathways we identified would provide new insights for the genetic and mechanism study of BD. In particular, the novel pathways we first identified such as 'Ubiquitin mediated proteolysis', 'Oocyte meiosis' and 'Metabolism of xenobiotics by cytochrome P450' would provide new perspectives to the following studies. These pathways need and also deserve further validation and replication to explore the pathogenic mechanism of BD.

\section{FOOTNOTES}

This work was supported by the National Natural Science Foundation of China (Grant No. 31401139), Strategic Priority Research Program (B) of the Chinese Academy of Sciences (Grant No. XDB02030002) and State Administration of Foreign Experts Affairs of Chinese Academy of Sciences (CAS/SAFEA) International Partnership Program for Creative Research Teams (Grant No. Y2CX131003).

Suhua Chang, Jinglu Wang, Kunlin Zhang and Jing Wang declare that they have no conflict of interest. This article does not contain any studies with human or animal subjects performed by the any of the authors. 
Table 2. Validated pathways by two groups of GWAS datasets or at least two PBA tools

\begin{tabular}{|c|c|c|c|c|c|}
\hline \multirow[t]{2}{*}{ KEGG ID } & \multirow[t]{2}{*}{ Pathway } & \multirow{2}{*}{$\begin{array}{l}\text { Supported } \\
\text { datasets }\end{array}$} & \multicolumn{3}{|l|}{ Supported tools ${ }^{a}$} \\
\hline & & & $\begin{array}{l}i \text {-GSEA4GWAS } \\
\text { v2 (FDR) }\end{array}$ & $\begin{array}{l}\text { GenGen } \\
\text { (FDR) }\end{array}$ & $\begin{array}{l}\text { SRT } \\
\text { (Empirical P) }\end{array}$ \\
\hline hsa00983 & Drug metabolism-other enzymes & Group A-Group B & $0.001(\mathrm{~A}), 0.0602(\mathrm{~B})$ & $0.025(\mathrm{~B})$ & \\
\hline hsa00830 & Retinol metabolism & Group A-Group B & $0.0025(\mathrm{~A}), 0.0438(\mathrm{~B})$ & $0.021(\mathrm{~B})$ & \\
\hline hsa00140 & Steroid hormone biosynthesis & Group A-Group B & $0.003(\mathrm{~A}), 0.1941(\mathrm{~B})$ & $0.043(\mathrm{~B})$ & \\
\hline hsa00860 & Porphyrin and chlorophyll metabolism & Group A-Group B & $0.003(\mathrm{~A}), 0.0658(\mathrm{~B})$ & $0.034(\mathrm{~B})$ & \\
\hline hsa00980 & $\begin{array}{l}\text { Metabolism of xenobiotics by } \\
\text { cytochrome p } 450\end{array}$ & Group A-Group B & $0.0077(\mathrm{~A}), 0.0357(\mathrm{~B})$ & $0.004(\mathrm{~B})$ & \\
\hline hsa00982 & Drug metabolism - cytochrome p450 & Group A-Group B & $0.0264(\mathrm{~A}), 0.1413(\mathrm{~B})$ & $0.019(\mathrm{~B})$ & \\
\hline hsa04722 & Neurotrophin signaling pathway & Group A-Group B & $0.0586(\mathrm{~A}), 0.1478(\mathrm{~B})$ & $0(\mathrm{~B})$ & \\
\hline hsa04914 & $\begin{array}{l}\text { Progesterone-mediated oocyte } \\
\text { maturation }\end{array}$ & Group A-Group B & $0.0632(\mathrm{~A}), 0.0665(\mathrm{~B})$ & $0.003(B)$ & \\
\hline hsa04350 & TGF beta signaling pathway & Group A-Group B & $0.1426(\mathrm{~A}), 0.1456(\mathrm{~B})$ & & \\
\hline hsa04960 & $\begin{array}{l}\text { Aldosterone-regulated } \\
\text { sodium reabsorption }\end{array}$ & Group A-Group B & $0.1501(\mathrm{~A}), 0.1383(\mathrm{~B})$ & $0.249(\mathrm{~B})$ & \\
\hline hsa05214 & Glioma & Group A-Group B & $0.1507(\mathrm{~A}), 0.1466(\mathrm{~B})$ & $0.108(B)$ & \\
\hline hsa05340 & Primary immunodeficiency & Group A-Group B & $0.2111(\mathrm{~A}), 0.2086(\mathrm{~B})$ & & $0.011(\mathrm{~A})$ \\
\hline hsa05217 & Basal cell carcinoma & Group B & $0.2146(\mathrm{~B})$ & $0(B)$ & \\
\hline hsa04114 & Oocyte meiosis & Group B & $0.043(B)$ & $0(B)$ & $0.007(\mathrm{~B})$ \\
\hline hsa04512 & ECM-receptor interaction & Group B & $0.1643(\mathrm{~B})$ & $0.001(\mathrm{~B})$ & \\
\hline hsa00510 & $\mathrm{N}$-glycan biosynthesis & Group B & $0.152(B)$ & $0.001(B)$ & \\
\hline hsa05412 & $\begin{array}{l}\text { Arrhythmogenic right ventricular } \\
\text { cardiomyopathy (ARVC) }\end{array}$ & Group B & $0.2058(\mathrm{~B})$ & $0.002(\mathrm{~B})$ & \\
\hline hsa00601 & $\begin{array}{l}\text { Glycosphingolipid biosynthesis } \\
\text { lacto and neolacto series }\end{array}$ & Group B & 0.1406 (B) & $0.002(\mathrm{~B})$ & \\
\hline hsa04540 & Gap junction & Group B & $0.166(B)$ & $0.003(\mathrm{~B})$ & \\
\hline hsa04720 & Long-term potentiation & Group B & $0.0482(\mathrm{~B})$ & $0.003(\mathrm{~B})$ & \\
\hline hsa04120 & Ubiquitin mediated proteolysis & Group B & $0.019(\mathrm{~B})$ & $0.002(\mathrm{~B})$ & $0.021(\mathrm{~B})$ \\
\hline hsa04115 & p53 signaling pathway & Group B & $0.109(\mathrm{~B})$ & $0.003(\mathrm{~B})$ & \\
\hline hsa04260 & Cardiac muscle contraction & Group B & $0.0456(\mathrm{~B})$ & $0.007(\mathrm{~B})$ & \\
\hline hsa02010 & $\mathrm{ABC}$ transporters & Group B & $0.1515(\mathrm{~B})$ & $0.008(\mathrm{~B})$ & \\
\hline hsa00040 & $\begin{array}{l}\text { Pentose and glucuronate } \\
\text { interconversions }\end{array}$ & Group B & $0.2056(\mathrm{~B})$ & $0.039(\mathrm{~B})$ & \\
\hline hsa05219 & Bladder cancer & Group B & $0.15(B)$ & $0.042(\mathrm{~B})$ & \\
\hline hsa03420 & Nucleotide excision repair & Group B & $0.1332(\mathrm{~B})$ & $0.046(B)$ & \\
\hline hsa04730 & Long-term depression & Group B & 0.1464 (B) & $0.058(\mathrm{~B})$ & \\
\hline hsa00591 & Linoleic acid metabolism & Group B & 0.1444 (B) & $0.111(\mathrm{~B})$ & \\
\hline hsa04110 & Cell cycle & Group B & & $0.001(\mathrm{~B})$ & $0.042(\mathrm{~B})$ \\
\hline hsa04142 & Lysosome & Group A & $0.2291(\mathrm{~A})$ & & $0.008(\mathrm{~A})$ \\
\hline hsa04070 & $\begin{array}{l}\text { Phosphatidylinositol } \\
\text { signaling system }\end{array}$ & Group A & $0.201(\mathrm{~A})$ & & $0.043(\mathrm{~A})$ \\
\hline hsa05221 & Acute myeloid leukemia & Group A & $0.2093(\mathrm{~A})$ & & $0.044(\mathrm{~A})$ \\
\hline
\end{tabular}

a (A) Denotes the FDR or Empirical P in Group A, (B) denotes the FDR or Empirical P in Group B. FDR < 0.05 in $i$-GSEA4GWAS v2 are bold. 
Suhua Chang ${ }^{1}$, Jinglu Wang ${ }^{1,2}$, Kunlin Zhang ${ }^{1}$, Jing Wang ${ }^{1 凶}$

${ }^{1}$ Key Laboratory of Mental Health, Institute of Psychology, Chinese Academy of Sciences, Beijing 100101, China

2 University of Chinese Academy of Sciences, Beijing 100049, China

$\bowtie$ Correspondence: wangjing@psych.ac.cn (J. Wang)

\section{OPEN ACCESS}

This article is distributed under the terms of the Creative Commons Attribution 4.0 International License (http://creativecommons.org/ licenses/by/4.0/), which permits unrestricted use, distribution, and reproduction in any medium, provided you give appropriate credit to the original author(s) and the source, provide a link to the Creative Commons license, and indicate if changes were made.

\section{REFERENCES}

Barnett JH, Smoller JW (2009) Neuroscience 164:331-343

Chang SH, Gao L, Li Z, Zhang WN, Du Y, Wang J (2013) Biol Psychiatry 74:727-733

Chen L, Zhang L, Zhao Y, Xu L, Shang Y, Wang Q, Li W, Wang H, Li $X$ (2009) Bioinformatics 25:237-242
Duncan LE, Holmans PA, Lee PH, O'Dushlaine CT, Kirby AW, Smoller JW, Ongur D, Cohen BM (2014) PLoS One 9:e89441

O'Dushlaine C, Kenny E, Heron EA, Segurado R, Gill M, Morris DW, Corvin A (2009) Bioinformatics 25:2762-2763

Peng G, Luo L, Siu H, Zhu Y, Hu P, Hong S, Zhao J, Zhou X, Reveille JD, Jin L et al (2010) Eur J Hum Genet 18:111-117

Shaltiel G, Chen G, Manji HK (2007) Curr Opin Pharmacol 7:22-26 Sklar P, Ripke S, Scott LJ, Andreassen OA, Cichon S, Craddock N, Edenberg HJ, Nurnberger JI Jr, Rietschel M, Blackwood D et al (2011) Nat Genet 43:977-983

Wang K, Li M, Bucan M (2007) Am J Hum Genet 81:1278-1283

Wang K, Li M, Hakonarson H (2010) Nat Rev Genet 11:843-854

Wellcome Trust Case Control Consortium (2007) Nature 447:661678

Welter D, MacArthur J, Morales J, Burdett T, Hall P, Junkins $H$, Klemm A, Flicek P, Manolio T, Hindorff L et al (2014) Nucleic Acids Res 42:D1001-D1006

Yu H, Bi W, Liu C, Zhao Y, Zhang D, Yue W (2014a) Prog Neuropsychopharmacol Biol Psychiatry 51:140-145

Yu H, Bi W, Liu C, Zhao Y, Zhang JF, Zhang D, Yue W (2014b) J Psychiatr Res 50:73-78

Zhang K, Chang S, Guo L, Wang J (2014) Protein Cell 5:258-260
Suhua Chang and Jinglu Wang contributed to this work equally.

Electronic supplementary material The online version of this article (doi:10.1007/s13238-015-0201-1) contains supplementary material, which is available to authorized users. 